\title{
Reflections on the Covariance of Modified Teleparallel Theories of Gravity
}

\author{
Cecilia Bejarano ${ }^{1}$, Rafael Ferraro ${ }^{1,2}$, Franco Fiorini ${ }^{3}$ and María José Guzmán ${ }^{4, *}$ \\ 1 Instituto de Astronomía y Física del Espacio (IAFE), CONICET, Universidad de Buenos Aires, Casilla de Correo \\ 67, Sucursal 28, Buenos Aires 1428, Argentina; cbejarano@iafe.uba.ar (C.B.); ferraro@iafe.uba.ar (R.F.) \\ 2 Departamento de Física, Facultad de Ciencias Exactas y Naturales, Universidad de Buenos Aires, \\ Ciudad Universitaria, Pabellón I, Buenos Aires 1428, Argentina \\ 3 Departamento de Ingeniería en Telecomunicaciones and Instituto Balseiro, Centro Atómico Bariloche, \\ Av. Ezequiel Bustillo 9500, San Carlos de Bariloche CP 8400, Argentina; francof@cab.cnea.gov.ar \\ 4 Departamento de Física y Astronomía, Facultad de Ciencias, Universidad de La Serena, Av. Juan Cisternas 1200 , \\ La Serena 1720236, Chile \\ * Correspondence: maria.j.guzman.m@gmail.com
}

Received: 23 May 2019; Accepted: 18 June 2019; Published: 25 June 2019

\begin{abstract}
We review the current status of the Lorentz covariance in teleparallel and modified teleparallel theories of gravity, and discuss the controversial features of the different approaches. We also revisit the issue of the remnant Lorentz gauge symmetries in $f(T)$ gravity.
\end{abstract}

Keywords: teleparallel gravity; $f(T)$ gravity; Lorentz invariance

\section{Introduction}

The dynamical equations of any physical theory can always be found to be invariant with respect to a group of transformations, commonly termed as a covariance group. The principle of covariance in general relativity refers to the invariance of the form of the equations of motion under differentiable coordinate transformations, also known as the diffeomorphism group [1]. In the context of gravity theories written in terms of frame fields (also known as vierbein or tetrads, in dimension four), there is an additional symmetry group related to the freedom of boosting or rotating such frames. Since tetrads can be considered as locally mapping spacetime points in the tangent space and vice versa, they possess additional free indices related to the tangent space. Global and local Lorentz transformations in the tangent space indices are then allowed; therefore it can be talked about an additional covariance under Lorentz transformations in these kinds of theories, and discuss whether the action and the dynamical equations are covariant. It is clear that this discussion does not appear in theories written in terms of the metric tensor, as they are formulated in Lorentz covariant form from first principles.

Theories of gravity written in terms of the tetrad field have been considered since the beginning of general relativity. Early attempts to include the tetrad and the spin connection as independent variables in a gravitational theory were considered in Einstein-Cartan gravity; these are gravitational theories in a Riemann-Cartan manifold with non-trivial curvature and torsion. On the other hand, it is possible to achieve a fully equivalent version of general relativity in the tetrad formalism through the consideration of a curvatureless spin connection. These theories are built in a Weitzenböck spacetime, and historically the simplest choice for the spin connection is the Weitzenböck one, which depends linearly on first-order derivatives of the tetrad field. This version of general relativity is known as the teleparallel 
equivalent of general relativity (TEGR), since the Weitzenböck connection has the property that it parallel transports the tetrad field along the manifold, giving an absolute notion of parallelism at a distance [2]. The Lagrangian of TEGR is invariant under global Lorentz transformations of the tetrad field; however, it is just pseudo-invariant if the Lorentz transformations become local, since it differs from the Einstein-Hilbert Lagrangian through a boundary term that is sensitive to local Lorentz transformations. This feature is not relevant for the equations of motion, as the boundary term is integrated out once in the action, giving a dynamics fully equivalent to general relativity (GR). In particular, in spite of the fact that the tetrad contains more components than the metric, TEGR only involves those degrees of freedom associated with the metric [3].

Modified teleparallel theories of gravity where the TEGR is the underlying framework are extensively being studied nowadays, with special emphasis in confronting the main problems in cosmology related to the early inflationary stage and the late accelerated expansion regimes. Nonlinear modifications of a Lorentzian pseudo-invariant Lagrangian are expected to break local Lorentz symmetries, and to harbor extra degrees of freedom [4]. Recently, several arguments have been put forward in favor of covariant versions of modified teleparallel gravity that drop the assumption of the Weitzenböck connection in favor of a more general inertial spin connection, with its own advantages and problems. The purpose of this article is to discuss the issues emerging from the peculiar behavior of teleparallel theories under local Lorentz transformations of the tetrad.

\section{Teleparallel Gravity}

\subsection{Geometric Foundations of General Relativity}

There are two main geometric objects that serve to build Lagrangians for gravitational theories: the torsion $\mathbf{T}^{a}$ and the curvature $\mathbf{R}_{b}^{a}$. The torsion is a set of 2 -forms defined as

$$
\mathbf{T}^{a}=D \mathbf{E}^{a}=d \mathbf{E}^{a}+\omega^{a}{ }_{b} \wedge \mathbf{E}^{b} .
$$

Here, the 1-forms $\mathbf{E}^{a}(x)$ make up the local basis of the cotangent space $\left(\mathbf{E}^{a}=E_{\mu}^{a} d x^{\mu}\right)$, and $d$ is the exterior derivative of forms. The set of 1 -forms $\omega^{a}{ }_{b}$ is the spin connection that provides the exterior derivative $D$ with a covariant character under local Lorentz transformations $\mathbf{E}^{a^{\prime}}=\Lambda^{a^{\prime}}{ }_{a}(x) \mathbf{E}^{a}$. This means that the tangent-space index in $\mathbf{T}^{a}$ is a Lorentz vector index, in the sense that $\mathbf{T}^{a}$ transforms like $\mathbf{E}^{a}$. For this, the spin connection $\omega^{a}{ }_{b}$ must transform as

$$
\omega_{b^{\prime}}^{a^{\prime}}=\Lambda_{a}^{a^{\prime}} \omega_{b}^{a} \Lambda_{b^{\prime}}^{b}+\Lambda_{a}^{a^{\prime}} d \Lambda_{b^{\prime \prime}}^{a}
$$

where $\Lambda_{a^{\prime}}^{a}$ is the respective inverse Lorentz transformation: $\Lambda_{a}^{a^{\prime}} \Lambda_{b^{\prime}}^{a}=\delta_{b^{\prime}}^{a^{\prime}}$.

On the other hand, the curvature $\mathbf{R}_{b}^{a}$ is a set of 2-forms that depends only on the spin connection, and reads

$$
\mathbf{R}_{b}^{a}=d \boldsymbol{\omega}_{b}^{a}+\boldsymbol{\omega}_{c}^{a} \wedge \boldsymbol{\omega}_{b}^{c} .
$$

Like the torsion, the curvature behaves as a Lorentz tensor under local Lorentz transformations of the basis.

The basis $\left\{\mathbf{e}_{a}\right\}$ of the tangent space $\left(\mathbf{e}_{a}=e_{a}^{\mu} \partial_{\mu}\right)$ is said dual to $\left\{\mathbf{E}^{a}\right\}$ if it fulfills $e_{a}^{\mu} E_{\mu}^{b}=\delta_{a}^{b}, e_{a}^{\mu} E_{v}^{a}=\delta_{v}^{\mu}$. In a space endowed with a metric tensor $\mathbf{g}$, it is useful to restrict the bases to be orthonormal:

$$
\mathbf{e}_{a} \cdot \mathbf{e}_{b}=g_{\mu \nu} e_{a}^{\mu} e_{b}^{v}=\eta_{a b}
$$


where $\eta_{a b}=\operatorname{diag}(1,-1,-1,-1)$ is the Minkowski tensor. The orthonormality condition allows for obtaining the metric from the tetrad; in fact, by using the duality, one obtains

$$
g_{\mu \nu}=\eta_{a b} E_{\mu}^{a} E_{v}^{b} .
$$

In addition, the non-metricity tensor $Q_{\lambda \mu \nu}=\nabla_{\lambda} g_{\mu \nu}$ involves the metric and the affine connection $\Gamma_{\nu \lambda}^{\mu}$, and sometimes is used as a third field to characterize the geometry and codify the gravitational interaction [5-8]. However, both general relativity and teleparallel gravity are based on metric connections, which are those that make $Q_{\lambda \mu \nu}=0$. This metricity requirement implies the validity of the Leibniz rule for the scalar product of vectors $\mathbf{a} \cdot \mathbf{b}=g_{\mu \nu} a^{\mu} b^{v}$. Therefore, in Equation (4), it results

$$
\left(\nabla_{\mathbf{e}_{c}} \mathbf{e}_{a}\right) \cdot \mathbf{e}_{b}+\mathbf{e}_{a} \cdot\left(\nabla_{\mathbf{e}_{c}} \mathbf{e}_{b}\right)=0,
$$

where $\nabla_{\mathbf{e}_{c}}=\nabla_{e_{c}^{\lambda} \partial_{\lambda}}=e_{c}^{\lambda} \nabla_{\lambda}$. The last equation implies a simple property of the spin connection, when written in an orthonormal basis. In fact, the affine connection and the spin connection are related in the following way: ${ }^{1}$

$$
\nabla_{\mathbf{e}_{c}} \mathbf{e}_{b}=\Gamma_{b c}^{a} \mathbf{e}_{a}, \quad \omega_{b}^{a}=\Gamma_{b c}^{a} \mathbf{E}^{c} \quad \text { or } \quad \Gamma_{b c}^{a}=\omega_{b}^{a}\left(\mathbf{e}_{c}\right) .
$$

Then, for the orthonormal basis of the previous equation, one gets

$$
0=\Gamma_{a c}^{d} \mathbf{e}_{d} \cdot \mathbf{e}_{b}+\mathbf{e}_{a} \cdot \Gamma_{b c}^{d} \mathbf{e}_{d}=\Gamma_{a c}^{d} \eta_{d b}+\Gamma_{b c}^{d} \eta_{a d},
$$

and multiplying by $\mathbf{E}^{c}$ :

$$
0=\omega_{a}^{d} \eta_{d b}+\omega_{b}^{d} \eta_{a d}=\omega_{b a}+\omega_{a b} .
$$

Thus, the metricity implies that the spin connection is anti-symmetric in an orthonormal basis. ${ }^{2}$ The condition of null torsion and metricity defines the Levi-Civita connection $\omega^{L}{ }_{b}$ in terms of first order derivatives of the tetrad, as a very well known formula. It will be convenient for what comes next to define an object representing the departure of $\omega_{b}^{a}$ from the Levi-Civita connection, which is a set of 1-forms called the contortion

$$
\mathbf{K}_{b}^{a}=\omega_{b}^{a}-\stackrel{L}{a}_{b} .
$$

$\mathbf{K}_{b}^{a}$ is a Lorentz tensor, since any difference of connections transforms as a tensor. Notice that $\mathbf{T}^{a}$ and $\mathbf{K}_{b}^{a}$ relates in a simple way (use $\stackrel{L}{\mathbf{T}}^{a}=0$ ):

$$
\mathbf{T}^{a}=\mathbf{K}_{b}^{a} \wedge \mathbf{E}^{b} .
$$

The common understanding of the gravitational phenomena assumes that its geometrization should be represented by the Levi-Civita spacetime curvature $\mathbf{R}^{a}{ }_{b}$. Thus, the Einstein-Hilbert Lagrangian is the 4 -form or volume form

$$
L_{E H}=\frac{1}{4 \kappa} \epsilon_{a b c d} \mathbf{E}^{a} \mathbf{E}^{b} \mathbf{R}^{c d}
$$

(we have suppressed the wedge product signs $\wedge$ to abbreviate the writing, but we must keep in mind that the 1-forms anti-commute). Here, the tetrad is assumed orthonormal, so meaning that a metric is involved

\footnotetext{
Other authors define the affine connection as $\nabla_{\mathbf{e}_{c}} \mathbf{e}_{b}=\Gamma_{c b}^{a} \mathbf{e}_{a}$.

The anti-symmetry of the spin connection implies that $D \eta_{a b}=d \eta_{a b}-\omega_{a}^{c} \eta_{c b}-\omega_{b}^{c} \eta_{a c}=-\omega_{b a}-\omega_{a b}=0$, and $D \varepsilon_{a b c d}=0$.
} 
in $L_{E H}$. Moreover, $L_{E H}$ is a Lorentz scalar. Thus, $L_{E H}$ is insensitive to local boosts and rotations of the tetrad; it is only sensitive to the metric.

We can use twice the definition of contortion (10) to relate the Levi-Civita curvature with the curvature belonging to an arbitrary connection:

$$
\stackrel{L}{R}^{c d}=\mathbf{R}^{c d}-d \mathbf{K}^{c d}-\mathbf{K}_{e}^{c} \omega^{e d}-\omega_{e}^{c} \mathbf{K}^{e d}+\mathbf{K}_{e}^{c} \mathbf{K}^{e d}=\mathbf{R}^{c d}-d \mathbf{K}^{c d}-\stackrel{L}{L}_{e} \mathbf{K}^{e d}-\mathbf{K}_{e}^{c} \omega^{L}-\mathbf{K}_{e}^{c} \mathbf{K}^{e d},
$$

where $-\mathbf{K}_{e}^{c} \stackrel{L}{e d}^{e d}={ }_{\omega^{e d}}^{L} \mathbf{K}_{e}^{c}=-{ }^{L}{ }^{d}{ }_{e} \mathbf{K}^{c e}$ (we have used the anti-commutativity and the metricity). Then, it results that

$$
\stackrel{L}{\mathbf{R}^{c d}}=\mathbf{R}^{c d}-\stackrel{L}{D} \mathbf{K}^{c d}-\mathbf{K}_{e}^{c} \mathbf{K}^{e d} .
$$

Therefore, the Einstein-Hilbert Lagrangian can be written as

$$
L_{E H}=\frac{1}{4 \kappa} \epsilon_{a b c d} \mathbf{E}^{a} \mathbf{E}^{b}\left(\mathbf{R}^{c d}-\stackrel{L}{D} \mathbf{K}^{c d}-\mathbf{K}_{e}^{c} \mathbf{K}^{e d}\right) .
$$

Since the Levi-Civita connection is torsionless, so $\stackrel{L}{D} \mathbf{E}^{a}=0$, we can recognize the boundary term $\stackrel{L}{D}\left(\epsilon_{a b c d} \mathbf{E}^{a} \mathbf{E}^{b} \mathbf{K}^{c d}\right)=d\left(\epsilon_{a b c d} \mathbf{E}^{a} \mathbf{E}^{b} \mathbf{K}^{c d}\right)$ (we have replaced the covariant exterior derivative with the exterior derivative since we are differentiating a Lorentz scalar). Therefore,

$$
L_{E H}=\frac{1}{4 \kappa} \epsilon_{a b c d} \mathbf{E}^{a} \mathbf{E}^{b}\left(\mathbf{R}^{c d}-\mathbf{K}_{e}^{c} \mathbf{K}^{e d}\right)-\frac{1}{4 \kappa} d\left(\epsilon_{a b c d} \mathbf{E}^{a} \mathbf{E}^{b} \mathbf{K}^{c d}\right) .
$$

\subsection{The Teleparallel Equivalent of General Relativity}

Today, it is widely spread that there are at least two other equivalent ways of codifying gravitational interactions: either by means of the torsion of spacetime or by its non-metricity. In the teleparallel approach, both the curvature and the non-metricity are zero; the gravitational phenomena are encoded in the torsion. Since the spacetime is curvatureless, then the parallel transport does not depend on the path; the parallelism is absolute. There is a simple way of building a teleparallel theory equivalent to general relativity (TEGR): since Equation (16) is valid whatever the connection is, we can take a curvatureless connection $\mathbf{R}^{c d}=0$ and dismiss the boundary term. Thus, we obtain

$$
L_{T E G R}=-\frac{1}{4 \kappa} \epsilon_{a b c d} \mathbf{E}^{a} \mathbf{E}^{b} \mathbf{K}_{e}^{c} \mathbf{K}^{e d} .
$$

This Lagrangian is quadratic in the Levi-Civita connection (i.e., it is quadratic in first derivatives of the tetrad), and contains a non-determined curvatureless spin connection $\omega^{a}{ }_{b}$. One could think that the theory itself should govern the connection. This way of thinking is motivated by general relativity, where the Levi-Civita connection can be obtained from varying the action independently with respect to the tetrad and the spin connection (Palatini formalism). In fact, even if the connection in Equation (12) was not chosen to be $\omega^{a}{ }_{b}$, we could still obtain it by varying the Lagrangian with respect to $\omega_{b}^{a}$ :

$$
\delta_{\omega} L_{E H}=\frac{1}{4 \kappa} \epsilon_{a b c d} \mathbf{E}^{a} \mathbf{E}^{b} \delta_{\omega} \mathbf{R}^{c d},
$$


where, for any metric connection, it is

$$
\delta_{\omega} \mathbf{R}^{c d}=d \delta \boldsymbol{\omega}^{c d}+\delta \boldsymbol{\omega}_{e}^{c} \omega^{e d}+\omega_{e}^{c} \delta \boldsymbol{\omega}^{e d}=d \delta \boldsymbol{\omega}^{c d}+\omega_{e}^{d} \delta \boldsymbol{\omega}^{c e}+\omega_{e}^{c} \delta \boldsymbol{\omega}^{e d}=D \delta \boldsymbol{\omega}^{c d} .
$$

Thus, dismissing boundary terms, we obtain

$$
\delta_{\omega} L_{E H}=-\frac{1}{4 \kappa} D\left(\epsilon_{a b c d} \mathbf{E}^{a} \mathbf{E}^{b}\right) \delta \boldsymbol{\omega}^{c d}=\frac{1}{2 \kappa} \epsilon_{a b c d} \mathbf{E}^{a} \mathbf{T}^{b} \delta \boldsymbol{\omega}^{c d},
$$

where we used $D \mathbf{E}^{b}=\mathbf{T}^{b}$. Thus, $\delta_{\omega} L_{E H}$ is zero if and only if the torsion is zero; therefore, the metric spin connection must be $\stackrel{L}{a}_{b}$.

On the other hand, the variation of $L_{T E G R}$ with respect to the connection,

$$
\delta_{\omega} L_{T E G R}=-\frac{1}{4 \kappa} \epsilon_{a b c d} \mathbf{E}^{a} \mathbf{E}^{b} \delta_{\omega}\left(\mathbf{K}_{e}^{c} \mathbf{K}^{e d}\right),
$$

where (we will use the metricity)

$$
\begin{aligned}
\epsilon_{a b c d} \delta_{\omega}\left(\mathbf{K}_{e}^{c} \mathbf{K}^{e d}\right) & =\epsilon_{a b c d} \delta_{\omega} \mathbf{K}_{e}^{c} \mathbf{K}^{e d}+\epsilon_{a b c d} \mathbf{K}_{e}^{c} \delta_{\omega} \mathbf{K}^{e d}=-\epsilon_{a b c d} \mathbf{K}^{e d} \delta_{\omega} \mathbf{K}_{e}^{c}+\epsilon_{a b c d} \mathbf{K}_{e}^{c} \delta_{\omega} \mathbf{K}^{e d} \\
& =-\epsilon_{a b d c} \mathbf{K}^{c c} \delta_{\omega} \mathbf{K}_{e}^{d}+\epsilon_{a b c d} \mathbf{K}_{e}^{c} \delta_{\omega} \mathbf{K}^{e d}=-\epsilon_{a b d c} \mathbf{K}_{e}^{c} \delta_{\omega} \mathbf{K}^{e d}+\epsilon_{a b c d} \mathbf{K}_{e}^{c} \delta_{\omega} \mathbf{K}^{e d} \\
& =2 \epsilon_{a b c d} \mathbf{K}_{e}^{c} \delta_{\omega} \mathbf{K}^{e d}
\end{aligned}
$$

leads to the result

$$
\delta_{\omega} L_{T E G R}=-\frac{1}{2 \kappa} \epsilon_{a b c d} \mathbf{E}^{a} \mathbf{E}^{b} \mathbf{K}_{e}^{c} \delta \boldsymbol{\omega}^{e d},
$$

where we used that $\delta_{\omega} \mathbf{K}^{e d}=\delta \boldsymbol{\omega}^{\text {ed }}$. Noticeably, the vanishing of $\delta_{\omega} L_{T E G R}$ will not lead to a curvatureless connection; on the contrary, it would lead to the Levi-Civita connection again, since the contortion is zero when $\omega^{c}{ }_{e}={\stackrel{L}{\omega^{c}}}_{e}$. This shows that the TEGR spin connection cannot be derived from $L_{T E G R}$. Instead, the curvatureless TEGR spin connection must be chosen, as it happens with the choice of the torsionless Levi-Civita connection in the metric formalism of general relativity. The conclusion is that $L_{E H}$ and $L_{T E G R}$ are equivalent from a metric point of view; i.e., they are equivalent when regarded as functionals of the tetrad.

Usually, the TEGR spin connection is chosen to be $\omega_{e}^{c}=0$ (Weitzenböck connection), which implies that the torsion is $\mathbf{T}^{a}=d \mathbf{E}^{a}$. Then, the components of the torsion are

$$
\mathbf{T}_{\mu \nu}^{a}=\partial_{\mu} E_{\nu}^{a}-\partial_{\nu} E_{\mu}^{a}, \quad \text { or } \quad T_{\mu \nu}^{\lambda}=e_{a}^{\lambda}\left(\partial_{\mu} E_{\nu}^{a}-\partial_{\nu} E_{\mu}^{a}\right) .
$$

The affine connection in a coordinate basis is ${ }^{3}$

$$
\Gamma_{\mu \nu}^{\lambda}=e_{a}^{\lambda} E_{\mu}^{b} E_{v}^{c} \Gamma_{b c}^{a}+e_{a}^{\lambda} \partial_{v} E_{\mu}^{a}=e_{a}^{\lambda} \partial_{\nu} E_{\mu}^{a} .
$$

However, in doing so, we have lost one of the fondest properties of the Lagrangian: if $\omega^{c}{ }_{e}$ is fixed to be zero, then $L_{T E G R}$ is no longer a Lorentz scalar. In fact, in Equation (17), $\mathbf{K}_{e}^{c}$ is a Lorentz tensor valued 1 -form whenever it is a difference of connections. However, if $\omega_{e}^{c}=0$, then $\mathbf{K}_{e}^{c}$ becomes the connection $\stackrel{L}{-\omega^{c}} e_{e}$; it is no longer a tensor under local Lorentz transformations. Therefore, $L_{T E G R}$ stops being a Lorentz

3 Notice that the coefficients $E_{\mu}^{a}, e_{a}^{\mu}$ are the links between anholonomous and coordinate basis. 
scalar. This loss of the invariance under local Lorentz transformations of the tetrad is not a problem at the level of the dynamical equations. Equation (16), where $L_{E H}$ is a Lorentz scalar, implies that, under local Lorentz transformations of the tetrad, $L_{T E G R}$ changes by a boundary term (pseudo-invariance). However, the loss of local Lorentz invariance will be a real hallmark on modified teleparallel theories like $f(T)$ gravity, which will lead to the appearance of extra degree(s) of freedom [4]. To avoid this annoying feature, it has been proposed that $\omega_{e}^{c}$ should not be zero, but belong to the family of the Lorentz transformations of $\omega_{b}^{a}=0$,

$$
\omega_{b}^{a}=\Lambda_{a^{\prime}}^{a} d \Lambda_{b}^{a^{\prime}}
$$

where $\Lambda$ represents any Lorentz transformation. This curvatureless form should not affect the metricity, since the requirement of metricity is tensorial in both coordinate indices and Lorentz (tangent space) indices. For instance, let us consider infinitesimal Lorentz transformations

$$
\Lambda_{a^{\prime}}^{a}=\delta_{a^{\prime}}^{a}-\frac{1}{2} \sigma^{g h}(x)\left(M_{g h}\right)_{a^{\prime}}^{a}+O\left(\sigma^{2}\right),
$$

where $\left(M_{g h}\right)_{a^{\prime}}^{a}$ are the (anti-symmetric) generators of the vector representation of the Lorentz group,

$$
\left(M_{g h}\right)_{a^{\prime}}^{a}=\delta_{g}^{a} \eta_{h a^{\prime}}-\delta_{h}^{a} \eta_{g a^{\prime}}
$$

and $\sigma^{g h}(x)$ are anti-symmetric parameters combining boosts and rotations in the transformation $\Lambda_{a^{\prime}}^{a}$. Then,

$$
\begin{aligned}
\boldsymbol{\omega}^{a b} & =\eta^{b c} \Lambda_{a^{\prime}}^{a} d \Lambda_{c}^{a_{c}^{\prime}}=-\frac{1}{2} \eta^{b c} \delta_{a^{\prime}}^{a}\left(\delta_{g}^{a^{\prime}} \eta_{h c}-\delta^{a^{\prime}} \eta_{g c}\right) d \sigma^{g h}+O\left(\sigma^{2}\right) \\
& =-\frac{1}{2}\left(\delta_{g}^{a} \delta_{h}^{b}-\delta_{h}^{a} \delta^{b}{ }_{g}\right) d \sigma^{g h}+O\left(\sigma^{2}\right)=-d \sigma^{a b}+O\left(\sigma^{2}\right) .
\end{aligned}
$$

Certainly, $\omega^{a b}=-d \sigma^{a b}$ is metric (although it is curvatureless only at the first order in $\sigma$ ).

The claims asserting that the choice (26) is the right choice for what is called "consistent teleparallel gravity" are based on the following argument: since TEGR is a theory that is pseudo-invariant under local Lorentz transformations, there is nothing special about the reference frame on which the spin connection vanishes, which would mean to put $\Lambda_{b^{\prime}}^{a}=\delta_{b^{\prime}}^{a}$ or any global Lorentz transformation, in (26). This is entirely true, although completely insubstantial at the level of TEGR. In fact, the spin connection is not a dynamical field in TEGR; TEGR is a dynamical theory just for the metric.

\section{Modified Teleparallel Gravities}

The traditionally used formulation of TEGR starts from the torsion scalar $T$ obtained when one replaces $\omega_{b}^{a}=0$ in Equation (17). After some calculation, one gets

$$
L_{T E G R}=\frac{1}{2 \kappa} E T d x^{0} \wedge d x^{1} \wedge d x^{2} \wedge d x^{3},
$$

where

$$
\begin{gathered}
T \equiv S_{\rho}{ }^{\mu v} T^{\rho}{ }_{\mu v}, \\
2 S_{\rho}{ }^{\mu v} \equiv K_{\rho}^{\mu v}+T_{\lambda}^{\lambda \mu} \delta_{\rho}^{v}-T_{\lambda}^{\lambda v} \delta_{\rho}^{\mu},
\end{gathered}
$$

and $K_{\rho}^{\mu v}$ results from the components of the contortion,

$$
K_{\rho}^{\mu \nu}=g^{v \lambda} e_{a}^{\mu} E_{\lambda}^{b} K_{b \mu}^{a}=\frac{1}{2}\left(T_{\rho}^{\mu \nu}-T_{\rho}^{\mu \nu}+T_{\rho}^{v \mu}\right) .
$$


The torsion scalar in (31) is a suitable starting point for modifications to general relativity. The main advantage of this approach is that nonlinear generalizations of the torsion scalar will always result in equations of motion of second order in the tetrad field, as $T$ is an object depending on only first order derivatives. However, linear modifications to TEGR have been known for a time. For instance, in [9], a gravitational action has been built combining three quadratic pieces associated with the three irreducible parts of the torsion tensor (vectorial, axial and traceless-symmetric). The coefficients of the linear combination are arbitrary, although they are constrained by the physics in the solar system. However, they acquire defined values when local Lorentz invariance of the theory is required, thus reducing it to TEGR. On the other hand, nonlinear modifications of TEGR in the form of arbitrary functions of the torsion scalar $T$, better known as $f(T)$ theories of gravity, have gained attention since they can describe an inflationary early expansion without resorting to an inflaton field [10]. In addition, they can mimic dark energy by providing a late time accelerated expansion of the universe [11] (for an extensive list of references, see [12]).

In $f(T)$ gravity, the action is

$$
I=\frac{1}{2 \kappa} \int E f(T) d^{4} x
$$

Varying this action with respect to the tetrad, the dynamical equations are obtained:

$$
4 e \partial_{\mu}\left(f^{\prime}(T) E e_{a}^{\lambda} S_{\lambda}^{\mu v}\right)+4 f^{\prime}(T) e_{a}^{\lambda} T_{\mu \lambda}^{\sigma} S_{\sigma}^{\mu v}-e_{a}^{v} f(T)=-2 \kappa e_{a}^{\lambda} \mathcal{T}_{\lambda}{ }^{v},
$$

where $\mathcal{T}_{\lambda}{ }^{v}$ is the energy-momentum tensor of matter coupled to the metric tensor. Originally, $f(T)$ gravity was conceived for dealing with strong space-time singularities, so high-energy deformations of the sort $f(T)=T+T^{2} / \lambda+O\left(1 / \lambda^{2}\right)$ were considered. For these models, $\lambda$ introduces the length scale $\lambda^{-1 / 2}$ at which local Lorentz invariance would no longer exist as a full symmetry, giving way to the remnant ones. For instance, in Ref. [10], the constant $\lambda^{1 / 2}$ plays the role of a maximum attainable Hubble factor in the context of spatially flat FRW cosmological models. In that case, the Big Bang singularity is replaced by an early de Sitter stage of a purely geometrical character in which the Hubble factor $H_{\max }=(\lambda / 12)^{1 / 2}$ drives the inflationary era. Much more recently [13], and in a quite different context, it was shown that the Schwarzschild curvature singularity is replaced by an infinitely long cosmic string with constant curvature invariants related to $\lambda$. In this case, the length scale is $2|\lambda|^{-1 / 2}$, and it completely changes the structure of the black hole interior by rendering the spacetime geodesically complete. In the cosmological example, the presence of the scale $H_{\max }$ manifests itself at very early times of the cosmic evolution, while, in the black hole interior, the length scale involves a constant curvature asymptotic region located well inside the event horizon. In both cases, the breaking of the Lorentz symmetry occurs in times or places very distant from our daily experience.

Since their introduction, $f(T)$ theories have revealed several subtleties concerning the appearance of preferred (proper) reference frames, encoded in the tetrad field $\mathbf{E}^{a}\left(x^{\mu}\right)$. Actually, it was clear since the early developments in the field that the local action of the Lorentz group on a given solution $\mathbf{E}^{a}(x)$ of the $f(T)$ motion equations leads to another tetrad $\mathbf{E}^{a^{\prime}}=\Lambda^{a^{\prime}}{ }_{a}(x) \mathbf{E}^{a}$, which is not generally a solution, even though both of them generate the same metric tensor $\mathbf{g}=\eta_{a b} \mathbf{E}^{a} \mathbf{E}^{b}$. This is basically because the equations of motion determine more degrees of freedom than those captured by the metric tensor [14]; some attempts have been made for capturing the number and nature of these degrees of freedom through Hamiltonian analysis [3,4], conformal transformations [15,16], cosmological perturbations [17-20], among others. The extra degree(s) of freedom define the space-time structure by means of a parallelization, which fixes the tetrad components modulo certain remnant symmetries associated with the specific solution under consideration [21]. 


\section{Covariance in Modified Teleparallel Gravity}

The covariance of the TEGR action is guaranteed at the level of the equations of motion, but the Lorentz breaking behavior of the surface term could be significant for physical quantities defined in the boundary, as black hole thermodynamics. This issue has been previously investigated [22,23]; however, in this section, our focus will be on discussing covariant versions of modified TEGR. The pseudo-invariant character of TEGR gravity echoes in nonlinear modifications (as $f(T)$ gravity) in the form of explicit Lorentz breaking at the level of the action and the equations of motion. This issue has been noticed from the very beginning [10], and it was firstly associated with the appearance of extra degrees of freedom encoded in the components of the tetrad field. From a theoretical point of view, covariance under local Lorentz symmetries is a desirable feature for any physical theory, but, at the experimental level, this symmetry breaking would not be detectable, as the metric tensor remains unchanged regardless of the privileged orientation of reference frames. However, the theoretical issue on the covariance of the Lagrangian formulation is part of an active discussion, and several opinions have been wielded in the literature. Our aim is to review some of them and contribute to the debate.

Early discussion on the covariantization of $T$ has been schematized in Ref. [24]. The authors consider the possibility of giving up the teleparallel restriction $\omega_{b v}^{a}=0$, and take the metric and the contortion as variables to be independently varied. They conclude that the $f(T)$ action is dynamically trivial for $K_{\mu \nu}^{\rho}$, since it does not contain derivatives of the contortion, and it is inconsistent if matter is added. The impossibility of obtaining a curvatureless connection from varying $T$ with respect to $K_{\mu \nu}^{\rho}$ has been analyzed above (see Equation (23) and below).

An alternative path has been discussed in Ref. [25], where the authors studied a $f(T)$ action where the covariance of $T$ is restored, since the "pure frame" approach with $\omega_{b v}^{a}=0$ is not a priori chosen. Instead, the curvatureless condition is enforced through Lagrange multipliers in the action. The advantage of an explicitly covariant Lagrangian is overshadowed by the introduction of a large amount of additional fields encoded in these Lagrange multipliers. Although in principle, in the TEGR case, they describe the same dynamics because they could be fully determined through the equations of motion, more research is needed to determine if the nonlinear generalization can describe equivalent physics as described by "pure tetrad" modified TEGR.

Regarding the covariant formulation in [26], the authors argue that, if the formulation of $f(T)$ gravity started from a covariant version of TEGR, then the equations of motion would acquire an additional term equal to $f^{\prime}(T) \omega_{a v}^{b} S_{b}^{v \mu}$. This additional term would allow for recovering the local Lorentz invariance (but notice that TEGR dynamics are locally invariant without needing this term!). To obtain this term, the authors explain that the spin connection (26) would add a boundary term to the TEGR Lagrangian (this property results from Equation (16)). Since no dynamical equations for the curvatureless spin connection would be obtained in such case, they invoke a physical criterion to choose it. They say that the spin connection must be "inertial", in the sense that, if gravity is turned off (i.e., if $\kappa \rightarrow 0$ ), then the torsion should vanish (so the torsion would contain just gravitational effects but not inertial ones). This strategy seems to spoil the purpose of covariantization because the spin connection is not dynamically determined, but it is found a posteriori for each geometry. For a skeptical mind, this strategy can be considered even more intricate than sticking to the simplest spin connection choice, that is the Weitzenböck one. Then, the spin connection becomes an ad hoc non-dynamical field that removes the undesired effects of an improper parallelization. Theoretical concerns about the formulation of this covariant approach in a univocal standard variational principle come up, in order to avoid introducing degrees of freedom by hand.

Some remarks on different possibilities of variational procedures in teleparallel gravities have been exposed in Ref. [27]. The case that is of relevance for $f(T)$ gravity is when the variation of the spin 
connection is performed in the inertial class. For achieving a consistent variational procedure, the authors argue that arbitrary Lorentz matrices $\Lambda_{b}^{a}(x)$ can be considered as the fundamental field instead of the spin connection. As a consequence, the TEGR Lagrangian can be regarded as a function of both the tetrad and the arbitrary Lorentz matrix, where the latter now acts as a physical field too. In the TEGR case, the variation of the spin connection (that is, the Lorentz matrix) is a harmless boundary term that vanishes under proper boundary conditions. In the $f(T)$ case, however, the variation on the spin connection indeed gives an additional equation, which reads

$$
T_{\mu \nu}^{\rho} \partial_{\rho} f^{\prime}(T)+T_{v} \partial_{\mu} f^{\prime}(T)-T_{\mu} \partial_{\nu} f^{\prime}(T)=0
$$

These are equivalent to the antisymmetric part of the equations of motion in the pure tetrad approach of $f(T)$ gravity, and its fulfillment is subject to a proper parallelization. This feature also appears in a wider class of modified teleparallel models [28]. In [29] and the recent review [30], Equation (36), written with an explicit dependence on the inertial spin connection as

$$
\partial_{\mu} f^{\prime}(T)\left[\partial_{\nu}\left(E e_{[a}^{\mu} e_{b]}^{v}\right)+2 E e_{c}^{[\mu} e_{[a}^{v]} \omega_{b] v}^{c}\right]=0,
$$

has been used in some examples, exhibiting the covariantization procedure. There are two issues for which we feel the need to provide additional discussion. The first point has to do with the fact that Equation (37), and the covariantization procedure, are only possible whenever the matter contribution in the rhs vanishes. Such contributions, coming from macroscopic spinorial fluids, have not been properly studied in the literature due to a lack of understanding in the correct spinorial coupling prescription. It is clear that the covariantization procedure is not general enough for including this interesting setup, and further research is encouraged in this direction, as it could be related with the physical interpretation of the additional degree(s) of freedom of the $f(T)$ theory.

Secondly, some concerns on the counting of degrees of freedom, and the first-class constraints that would generate gauge transformations for the inertial spin connection, have been briefly exposed in [31] (although their criticism is mainly directed to the covariant TEGR procedure). This resonates with our previous thoughts, and these concerns are also relevant for the covariantization procedure in $f(T)$ gravity. Nonetheless, it is relevant to mention that recent work on the Hamiltonian formalism for the so-called "new general relativity" has attempted to include the inertial spin connection in the formalism [32]. Their claim is that the inertial choice in new general relativity represents pure gauge degrees of freedom, and, consequently, the Weitzenböck connection is suitable for developing the Hamiltonian analysis (which, of course, tremendously simplifies the Poisson brackets calculations). It is clear that the consistency of this approach needs to be revisited, but, equally important, to analyze if these claims have something to say concerning the issue of the degree(s) of freedom of $f(T)$ and teleparallel gravity extensions.

\section{Remnant Symmetries and The Lorentz Group}

A simple example will help us to fathom some of the subtleties that lie behind the system (35). Let us consider the easiest solution of the vacuum field equations, which should correspond to the absence of gravity. Thus, let us consider Minkowski spacetime (Min), and take the following tetrad field

$$
\mathbf{E}^{0}=d t, \quad \mathbf{E}^{1}=d r, \quad \mathbf{E}^{2}=r d \theta, \quad \mathbf{E}^{3}=r \sin \theta d \phi,
$$


which is consistent with the line element $d s^{2}=d t^{2}-d r^{2}-r^{2}\left(d \theta^{2}+\sin ^{2} \theta d \phi^{2}\right)$ describing Min in spherical coordinates $(t, r, \theta, \phi)$. For the above field, we have $T=2 r^{-2}$, and the equations reduce to the system

$$
\begin{aligned}
16 f_{T T}-2 r^{2} f_{T}+f r^{4} & =0 \\
8 f_{T T}-2 r^{2} f_{T}+f r^{4} & =0 \\
-2 r^{2} f_{T}+f r^{4} & =0 \\
f_{T T} & =0
\end{aligned}
$$

where $f_{T T}=f^{\prime \prime}(T), f_{T}=f^{\prime}(T)$, and $f=f(T)$. It is clear that the only solution to this system involves $f(T) \propto T$, which is just GR. Does it mean that Min is not a solution of vacuum $f(T)$ gravity? What is exactly the problem concerning the frame (38)? Let us suppose that, instead of (38), we start from

$$
\mathbf{E}^{0}=d t, \quad \mathbf{E}^{1}=d \rho, \quad \mathbf{E}^{2}=\rho d \phi, \quad \mathbf{E}^{3}=d z
$$

which leads to the Minkowskian line element $d s^{2}=d t^{2}-d \rho^{2}-\rho^{2} d \phi^{2}-d z^{2}$ written in cylindrical coordinates $(t, \rho, \phi, z)$. It is simple to check that the Weitzenböck invariant vanishes for the tetrad (40), and the vacuum field Equation (35) take the remarkably simple form $f(T)=0$. This means that any ultraviolet deformation of GR (i.e., any function $f(T)$ verifying $f(0)=0$ and $f_{T}(0)=1$ ) admits Min as a vacuum solution through the fields (40).

Finally, consider the (Euclidean) tetrad field

$$
\mathbf{E}^{0}=d t, \quad \mathbf{E}^{1}=d x, \quad \mathbf{E}^{2}=d y, \quad \mathbf{E}^{3}=d z,
$$

which, of course, give us the standard Cartesian line element $d s^{2}=d t^{2}-\delta_{\alpha \beta} d x^{\alpha} d x^{\alpha}$. Again, $T=0$ and the equations of motion are $f(T)=0$, which are automatically fulfilled for any $\operatorname{UV} f(T)$ deformation of GR.

The point behind the results just obtained (the distinction between different frames leading to a certain metric tensor) naturally extends to a general spacetime $(\mathcal{M}, \mathbf{g}(x))$, or, more precisely, $\left(\mathcal{T}^{\star} \mathcal{M}, \mathbf{E}^{a}(x)\right)$. We would like to raise the following points:

1. Why, for a given spacetime $\left(\mathcal{T}^{\star} \mathcal{M}, \mathbf{E}^{a}(x)\right)$, there are certain proper tetrads $\mathbf{E}^{a}$, and others that definitely do not lead to a consistent set of equations of motion for a function $f(T)$ other than the one corresponding to GR?

2. Once the above point was established, is there any way of counting the number of proper tetrads, and some systematic procedure in order to obtain them?

3. What is the physical meaning of the proper tetrads?

In relation to the first point, and coming back to the example concerning Minkowski space, we can find the answer in the topological structure of the different frames involved. Of course, the manifold topology of Min is just $R^{4}$, and this is independent from the chosen tetrad. However, at the level of the vector fields $\mathbf{e}_{a}$, things are different; in the case of the frame (38), we see that every field in the family points at one of the directions defined by the spherical coordinates lines, and not mixed components appear. This means that, as far as the dual vector fields concerns, the topology they are actually seeing is $R^{2} \times S^{2}$. However, the diagonal form of the frame implies that the fields $\mathbf{E}^{2}$ and $\mathbf{E}^{3}$ are vector fields covering the whole tangent space of the 2-sphere, which is impossible because the 2-sphere is not parallelizable (spherically symmetric solutions with the same feature have been reported in [33]). Of course, the topological space $R^{2} \times S^{2}$ is parallelizable, just think about it as $R \times R \times S^{2}$, which is a product of the parallelizable manifolds $R$ and $R \times S^{2}$. Nevertheless, the global, everywhere non null, smooth vectors fields covering the tangent space of $R^{2} \times S^{2}$, are far from having the simple diagonal structure of (38)—see Equation (42) below. 
In the case of the frame (40), the situation is considerably different. Even though every field in the family is also pointing at the directions defined by the cylindrical coordinates lines, the topology they are defining is now $R^{3} \times S^{1}$. The diagonal structure suggests that the parallelization of the entire set is obtained as a topological product of parallelizable submanifolds, which is correct in this case due to the fact that $R$ and $S^{1}$ are parallelizable by themselves. This is the reason why the fields (40) constitute a proper tetrad for describing Min.

Finally, the frame (41) also represents a proper tetrad. Actually, it should be considered as the canonical, more natural parallelization of Min because the topology defined by the (Cartesian) set of vector fields coinciding with one of the latter. Note that both proper frames (40) and (41) give rise to the same null value of the Weitzenböck invariant $T$. This is not a coincidence.

An important observation concerning the role of the local coordinates would be adequate. Even though we are adopting local coordinates in order to write explicit forms of the vector fields, they are not essential at all. Once we have obtained a proper tetrad in a given coordinate system, we can change coordinates freely. For instance, nothing prevents us from finding a proper tetrad for Min in spherical coordinates; just take (40) or (41), and change coordinates accordingly; we will find $E_{\mu^{\prime}}^{a}=\partial x^{\mu} / \partial x^{\mu^{\prime}} E_{\mu^{\prime}}^{a}$, where $x^{\mu}$ refers to the cylindrical or Cartesian chart (depending on the case), and $x^{\mu^{\prime}}$ to the spherical one. If we start from the canonical frame (41), we will get

$$
\begin{aligned}
& \mathbf{E}^{0}=d t, \\
& \mathbf{E}^{1}=\sin \theta \cos \phi d r+r \cos \theta \cos \phi d \theta-r \sin \theta \sin \phi d \phi, \\
& \mathbf{E}^{2}=\sin \theta \sin \phi d r+r \cos \theta \sin \phi d \theta+r \sin \theta \cos \phi d \phi, \\
& \mathbf{E}^{3}=\cos \theta d r-r \sin \theta d \theta .
\end{aligned}
$$

Clearly, a parallelization of Min in spherical coordinates is quite more involved than the (incorrect) choice (38). Take note that the block structure in (42) emphasizes the fact that the spatial part of the frame is actually a parallelization of the submanifold $R \times S^{2}$.

\section{The Remnant Group}

We devote this section to the second point raised above. A systematic approach to the study of the proper frames describing a certain spacetime $\left(\mathcal{T}^{\star} \mathcal{M}, \mathbf{E}^{a}(x)\right)$ within the context of $f(T)$ gravity involves the characterization of the remnant group of Lorentz transformations of $\left(\mathcal{T}^{\star} \mathcal{M}, \mathbf{E}^{a}(x)\right)$. Details of the following exposition can be found in [21].

In general, under a Lorentz transformation of the tetrad $\mathbf{E}^{a} \rightarrow \mathbf{E}^{a^{\prime}}=\Lambda^{a^{\prime}}{ }_{a} \mathbf{E}^{a}$, the torsion scalar $T$ transform is as follows:

$$
T \boldsymbol{\Omega} \rightarrow T^{\prime} \boldsymbol{\Omega}=T \mathbf{\Omega}+d\left(\epsilon_{a b c d} \mathbf{E}^{a} \wedge \mathbf{E}^{b} \wedge \eta^{d e} \Lambda_{f^{\prime}}^{c} d \Lambda_{e}^{f^{\prime}}\right)
$$

$\left(\boldsymbol{\Omega}=E d x^{0} \wedge d x^{1} \wedge d x^{2} \wedge d x^{3}\right.$ is the spacetime volume), so the torsion scalar is not really a Lorentz scalar. The remnant group $\mathcal{A}\left(\mathbf{E}^{a}\right)$ of a given spacetime $\left(\mathcal{T}^{\star} \mathcal{M}, \mathbf{E}^{a}(x)\right)$ is defined as the subgroup of $\operatorname{SO}(1,3)$ under which $T$ becomes a Lorentz scalar, i.e., by demanding

$$
d\left(\epsilon_{a b c d} \mathbf{E}^{a} \wedge \mathbf{E}^{b} \wedge \eta^{d e} \Lambda_{f^{\prime}}^{c} d \Lambda_{e}^{f^{\prime}}\right)=0
$$


Of course, the global Lorentz group $\left(d \Lambda_{e}^{f^{\prime}}=0\right)$ is always included in $\mathcal{A}\left(\mathbf{E}^{a}\right)$, for all $\mathbf{E}^{a}$. For infinitesimal transformations, we have the expression (27), where the matrices $M_{g h}$ given in Equation (28) are boosts generators, $K_{\alpha}=M_{0 \alpha}$, and rotation generators $J_{\alpha}=-\frac{1}{2} \epsilon_{\alpha \beta \gamma} M^{\beta \gamma}$. The Lorentz algebra reads

$$
\left[J_{\alpha}, J_{\beta}\right]=\epsilon_{\alpha \beta \gamma} J^{\gamma}, \quad\left[K_{\alpha}, K_{\beta}\right]=-\epsilon_{\alpha \beta \gamma} J^{\gamma}, \quad\left[K_{\alpha}, J_{\beta}\right]=\epsilon_{\alpha \beta \gamma} K^{\gamma} .
$$

It is straightforward to show that Equation (44) at the infinitesimal level reduces to

$$
\epsilon_{a b c d} d\left(\mathbf{E}^{a} \wedge \mathbf{E}^{b}\right) \wedge d \sigma^{c d}=0
$$

At this point, it results in being very convenient to introduce a useful concept with regard to the solutions of Equation (46). A sufficient condition for a given element of $S O(3,1)$ to belong to $\mathcal{A}\left(E^{a}\right)$ comes when one classifies the solutions $\mathbf{E}^{a}$ of the equations of motion (35) according to the number of closed 2-forms they involve. A solution $\left\{\mathbf{E}^{a}\right\}$ will be called an $n$-closed-area frame (n-CAF), if it satisfies $d\left(\mathbf{E}^{a} \wedge \mathbf{E}^{b}\right)=0$ for $n$ of the six different pairs $(a, b)$. The utility of this definition can be seen through a couple of simple examples.

Let us suppose that a given solution has the 1-CAF defined by the 2-form $\mathbf{E}^{0} \wedge \mathbf{E}^{1}$. As $d\left(\mathbf{E}^{0} \wedge \mathbf{E}^{1}\right)=0$, the local parameter $\sigma^{23}$ is totally free in Equation (46), which means that $M_{23} \equiv J_{1}$ can be freely chosen. In other words, we have that $\operatorname{Rot}_{x^{1}} \subset \mathcal{A}\left(E^{a}\right)$ (here $\operatorname{Rot}_{x^{1}}$ stands for the subgroup of rotations about the $x^{1}$-axis). Albeit $\left\{\mathbf{E}^{a}\right\}$ is only a 1-CAF, other remnant symmetries could be allowed. Imagine that

$$
d\left(\mathbf{E}^{1} \wedge \mathbf{E}^{2}\right) \propto d t \wedge d x^{1} \wedge d x^{2} .
$$

This means that a Lorentz boosts $\sigma^{03}\left(t, x^{1}, x^{2}\right)$ will also be in $\mathcal{A}\left(E^{a}\right)$ (note the restriction on the coordinates in the boost generator). This is so because the 1-form $d \sigma^{03}$ in Equation (46) does not contain a term proportional to $d x^{3}$, so the wedge product $d\left(\mathbf{E}^{1} \wedge \mathbf{E}^{2}\right) \wedge d \sigma^{03}=0$. In this way, not only are the rotations about the $x^{1}$-axis in $\mathcal{A}\left(E^{a}\right)$, but also local (restricted) Lorentz boosts along the $x^{3}$-direction are admissible.

The structure of $\mathcal{A}\left(E^{a}\right)$ becomes richer as the number of closed areas approach its maximum value $n=6$. In general, for an $n$-CAF, we should expect $n$ one-parameter subgroups, but two-parameter Abelian subgroups could also exist for $n \geq 2$; take, for instance, the 2-CAF defined by $d\left(\mathbf{E}^{0} \wedge \mathbf{E}^{3}\right)=d\left(\mathbf{E}^{1} \wedge \mathbf{E}^{2}\right)=0$. This means that the remnant group will include local transformations generated by combinations of $M_{12}$ and $M_{03}$, which lead us two the 2-parameter Abelian group $\left\{J_{3}, K_{3}\right\}$. More details on the structure of $\mathcal{A}\left(E^{a}\right)$ can be consulted in [21].

Before proceeding to show specific examples, we should mention the following crucial fact; according to Equation (46), if $\left\{\mathbf{E}^{a}\right\}$ is a 6-CAF, then we get the maximum remnant symmetry because all the infinitesimal parameters $\sigma^{a b}$ remain free. Then, we obtain

$$
S O(3,1)_{\text {inf }} \subset \mathcal{A}\left(E^{a}\right),
$$

where $S O(3,1)_{\text {inf }}$ represents the infinitesimal Lorentz group. This result will be of utmost importance concerning the physical interpretation of $\mathcal{A}\left(E^{a}\right)$.

Moving on now to a more global level, let us study the importance of $\mathcal{A}\left(E^{a}\right)$ in solutions describing cosmological models. Let us consider anisotropic, homogeneous Bianchi type I spacetimes given by the line element

$$
d s^{2}=d t^{2}-a_{1}^{2}(t) d x^{2}-a_{2}^{2}(t) d y^{2}-a_{3}^{2}(t) d z^{2},
$$


where we used Euclidian coordinates $(t, x, y, z)$ and $a_{i}(t)$ are the scale factors. Metric (49) contains the important case $a_{1}=a_{2}=a_{3}$ representing a spatially flat FRW model. The topology of $(\mathcal{M}, \mathbf{g}(x))$ is $R^{4}$, then a canonical parallelization can be easily obtained by means of

$$
\mathbf{E}^{0}=d t, \quad \mathbf{E}^{1}=a_{1}(t) d x, \quad \mathbf{E}^{2}=a_{2}(t) d y, \quad \mathbf{E}^{3}=a_{3}(t) d z .
$$

Some clue on the structure of $\mathcal{A}\left(E^{a}\right)$ can be obtained by realizing that $d\left(\mathbf{E}^{0} \wedge \mathbf{E}^{\alpha}\right)=0, \forall \alpha$, so the tetrad (50) is a 3-CAF. This automatically implies that any local rotation $\sigma^{\beta \gamma}(x)$ of the frame (50) will be an admissible symmetry. This is so because, for every pair $(0, \alpha)$, we have a pair $(\beta, \gamma)(\alpha \neq \beta \neq \gamma)$, and there are three such pairs. Thus, despite the fact that metric (49) depends solely on time, proper tetrads depending on all the coordinates can be obtained by locally rotating the canonical frame (50).

Curiously enough, there are many more remnant symmetries present in this spacetime. Even though $\mathbf{E}^{\alpha} \wedge \mathbf{E}^{\beta}$ is not closed, we have

$$
d\left(\mathbf{E}^{\alpha} \wedge \mathbf{E}^{\beta}\right)=\left(a_{\alpha} a_{\beta}\right), t d t \wedge d x^{\alpha} \wedge d x^{\beta},
$$

where $\alpha, \beta$ are fixed indices. This is a nice physical example of additional (restricted) symmetries (see the discussion following Equation (47) in the previous section). As explained opportunely, the restriction on the spatial coordinates comes from the boost generator $\sigma^{0 \gamma}\left(t, x^{\alpha}, x^{\beta}\right), \alpha \neq \beta \neq \gamma$. Because of this, the structure of $\mathcal{A}\left(E^{a}\right)$ is very rich and includes the two-parametric Abelian groups

$$
\begin{aligned}
& \left\{K_{x}(t, y, z), J_{x}\left(x^{\mu}\right)\right\}, \\
& \left\{K_{y}(t, x, z), J_{y}\left(x^{\mu}\right)\right\}, \\
& \left\{K_{z}(t, x, y), J_{z}\left(x^{\mu}\right)\right\},
\end{aligned}
$$

where it is not in vain to emphasize again the coordinate restriction on the boost generators. The conclusions just obtained are also valid for the spatially flat FRW models, which seem to be in good agreement with the experimental facts concerning the large scale structure of the Universe.

Considering the nature of the space in question, we see that the remnant symmetries still constitute a vast, overrated set. In any physical cosmological model, it is always assumed that non-privileged positions exist; this assumption is of course supported by the fact that the Universe seems to look the same by looking at any direction, and nothing seems to indicate that this is valid only for special points in it, us between them. Of course, local irregularities such as stars or galaxies exist here and there, but these are considered tiny particles that are distributed in an approximately isotropic and homogeneous way throughout space. Thus, why bother with transformations depending on spatial coordinates? Why even consider them if here and there are basically the same? In other words, why demand local symmetries that are not consistent with the type of physical situations we are trying to model?

In this sense, the remnant symmetries are representative of the solution under consideration, and not general properties of all spaces. A cosmological spatially flat FRW model, for instance, is a physical theory about the temporal evolution of the scale factor $a(t)$, or, more properly, about the Hubble rate $H(t)$. A pair of observers connected by Lorentz transformations should be able to measure, let us say, the value of $H$. This will enable them to infer, by means of the equations of motions, physical properties of the Universe, as the distribution of energy-matter. However, from the outset, the theory predicts (for a given moment of time) the same value of $H(t)$ irrespective of the spatial position of the observers. Thus, only time dependent boost or rotations connecting physical observers should be considered as strictly physical symmetries in this specific model. As shown in Equation (52), $\mathcal{A}\left(E^{a}\right)$ includes these transformations. Any other dependence of the local parameters is simply asking too much. 


\section{Conclusions}

We have summarized several arguments about covariance in modified teleparallel gravities. There are still several issues to be addressed, in particular regarding the coupling of the antisymmetric part of the equations of motion of modified teleparallel to spinorial sources. It is of utmost importance to obtain a variational principle for this approach. Considerations about the inheritance of a remnant symmetry group of local Lorentz transformations have been discussed, which present an intriguing structure.

Author Contributions: Conceptualization: C.B., R.F., F.F. and M.J.G.; Investigation: C.B., R.F., F.F. and M.J.G.; Supervision: R.F.; Writing-original draft: C.B., R.F., F.F. and M.J.G.

Funding: This work was partially supported by the Consejo Nacional de Investigaciones Científicas y Técnicas (CONICET), Universidad de Buenos Aires and Instituto Balseiro (UNCUYO). M.J.G. has been funded by CONICYT-FONDECYT Postdoctoral grant No. 3190531. C.B., R.F., and F.F. are members of Carrera del Investigador Científico.

Acknowledgments: The authors thank Christian G. Boehmer, Alan A. Coley, Alexey Golovnev, Manuel Hohmann, Robert J. van den Hoogen and Martin Krššák for comments and helpful discussion. C.B. and M.J.G. are grateful to the organizers of the conference "Teleparallel Universes in Salamanca" for the kind invitation.

Conflicts of Interest: The authors declare no conflict of interest.

\section{References}

1. Sundermeyer, K. Symmetries in Fundamental Physics; Springer International Publishing: Berlin, Germany, 2014.

2. Aldrovandi, R.; Pereira, J.G. Teleparallel Gravity; Springer: Dordrecht, The Netherlands, 2013; Volume 173.

3. Ferraro, R.; Guzmán, M.J. Hamiltonian formulation of teleparallel gravity. Phys. Rev. D 2016, $94,104095$. [CrossRef]

4. Ferraro, R.; Guzmán, M.J. Hamiltonian formalism for $f(T)$ gravity. Phys. Rev. D 2018, 97, 104028. [CrossRef]

5. Adak, M.; Sert, O. A Solution to symmetric teleparallel gravity. Turk. J. Phys. 2005, 29, 1-7.

6. Adak, M.; Kalay, M.; Sert, O. Lagrange formulation of the symmetric teleparallel gravity. Int. J. Mod. Phys. D 2006, 15, 619-634. [CrossRef]

7. Beltrán Jiménez, J.; Heisenberg, L.; Koivisto, T. Coincident general relativity. Phys. Rev. D 2018, $98,044048$. [CrossRef]

8. Beltrán Jiménez, J.; Heisenberg, L.; Koivisto, T. The geometrical trinity of gravity. arXiv 2019, arXiv:1903.06830.

9. Hayashi, K.; Shirafuji, T. New general relativity. Phys. Rev. D 1979, 19, 3524.

10. Ferraro, R.; Fiorini, F. Modified teleparallel gravity: Inflation without inflaton. Phys. Rev. D 2007, 75, 084031. [CrossRef]

11. Bengochea, G.; Ferraro, R. Dark torsion as the cosmic speed up. Phys. Rev. D 2009, 79, 124019. [CrossRef]

12. Cai, Y.F.; Capozziello, S.; De Laurentis, M.; Saridakis, E.N. $f(T)$ teleparallel gravity and cosmology. Rep. Prog. Phys. 2016, 79, 106901. [CrossRef] [PubMed]

13. Boehmer, C.G.; Fiorini, F. The regular black hole in four dimensional Born Infeld gravity. Class. Quant. Grav. 2019, 36, 12LT01. [CrossRef]

14. Ferraro, R.; Guzmán, M.J. Quest for the extra degree of freedom in $f(T)$ gravity. Phys. Rev. D 2019, 98, 124037. [CrossRef]

15. Yang, R.J. Conformal transformation in $f(T)$ theories. Eur. Phys. Lett. 2011, 93, 60001. [CrossRef]

16. Wright, M. Conformal transformations in modified teleparallel theories of gravity revisited. Phys. Rev. D 2016, 93, 103002. [CrossRef]

17. Chen, S.H.; Dent, J.B.; Dutta, S.; Saridakis, E.N. Cosmological perturbations in $f(T)$ gravity. Phys. Rev. D 2011, 83, 023508. [CrossRef]

18. Li, B.; Sotiriou, T.P.; Barrow, J.D. Large-scale structure in $f(T)$ gravity. Phys. Rev. D 2011, 83, 104017. [CrossRef]

19. Izumi, K.; Ong, Y.C. Cosmological perturbation in $f(T)$ gravity revisited. J. Cosmol. Astropart. Phys. 2013, 1306, 029. [CrossRef] 
20. Golovnev, A.; Koivisto, T. Cosmological perturbations in modified teleparallel gravity models. J. Cosmol. Astropart. Phys. 2018, 1811, 012. [CrossRef]

21. Ferraro, R.; Fiorini, F. Remnant group of local Lorentz transformations in $f(T)$ theories. Phys. Rev. D 2015, 91, 064019. [CrossRef]

22. Krššák, M.; Pereira, J.G. Spin Connection and Renormalization of Teleparallel Action. Eur. Phys. J. C 2015, 75, 519. [CrossRef]

23. Krššák, M. Holographic renormalization in teleparallel gravity. Eur. Phys. J. C 2017, 77, 44. [CrossRef]

24. Sotiriou, T.P.; Li, B.; Barrow, J.D. Generalizations of teleparallel gravity and local Lorentz symmetry. Phys. Rev. D 2011, 83, 104030. [CrossRef]

25. Ong, Y.C.; Nester, J.M. Counting components in the lagrange multiplier formulation of teleparallel theories. Eur. Phys. J. C 2018, 78, 568. [CrossRef]

26. Krššák, M.; Saridakis, E.N. The covariant formulation of $f(T)$ gravity. Class. Quant. Grav. 2016, $33,115009$. [CrossRef]

27. Golovnev, A.; Koivisto, T.; Sandstad, M. On the covariance of teleparallel gravity theories. Class. Quant. Grav. 2017, 34, 145013. [CrossRef]

28. Hohmann, M.; Järv, L.; Krššák, M.; Pfeifer, C. Teleparallel theories of gravity as analogue of nonlinear electrodynamics. Phys. Rev. D 2018, 97, 104042. [CrossRef]

29. Hohmann, M.; Järv, L.; Ualikhanova, U. Covariant formulation of scalar-torsion gravity. Phys. Rev. D 2018, 97, 104011. [CrossRef]

30. Krššák, M.; Van Den Hoogen, R.J.; Pereira, J.G.; Boehmer, C.G.; Coley, A.A. Teleparallel theories of gravity: Illuminating a fully invariant approach. arXiv 2018, arXiv:1810.12932.

31. Maluf, J.W.; Ulhoa, S.C.; da Rocha-Neto, J.F. Difficulties of Teleparallel Theories of gravity with local Lorentz symmetry. arXiv 2018, arXiv:1811.06876.

32. Blixt, D.; Hohmann, M.; Pfeifer, C. Hamiltonian and primary constraints of new general relativity. Phys. Rev. D 2019, 99, 084025. [CrossRef]

33. Hohmann, M.; Järv, L.; Krššák, M.; Pfeifer, C. Modified teleparallel theories of gravity in symmetric spacetimes. arXiv 2019, arXiv:1901.05472.

(C) 2019 by the authors. Licensee MDPI, Basel, Switzerland. This article is an open access article distributed under the terms and conditions of the Creative Commons Attribution (CC BY) license (http:/ / creativecommons.org/licenses/by/4.0/). 